\title{
Evaluación de la sensibilidad diagnóstica de tres técnicas de laboratorio para la infección por influenza A: inmunocromatografía, IFD e IFD con citocentrifugado versus RPC-TR
}

\author{
Jan Wilhelm, Joao Yubero, Constanza Fuentes, Paulina Ríos, Oscar Leyton y Felipe Reyes
}

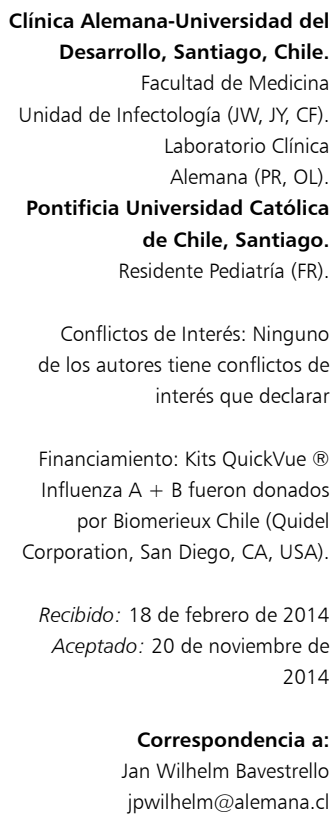

Clínica Alemana-Universidad del Desarrollo, Santiago, Chile. Facultad de Medicin Unidad de Infectología (JW, JY, CF). Laboratorio Clínica Alemana (PR, OL). Pontificia Universidad Católica de Chile, Santiago. Residente Pediatría (FR)

Conflictos de Interés: Ninguno de los autores tiene conflictos de interés que declarar

Financiamiento: Kits QuickVue ${ }^{\circledR}$ Influenza A + B fueron donados por Biomerieux Chile (Quide Corporation, San Diego, CA, USA)

Recibido: 18 de febrero de 2014 Aceptado: 20 de noviembre de

\section{Evaluation of three laboratory methods diagnostic sensitivity in influenza A infection: RIDT, DFA and DFA with cytocentrifugation versus RT-PCR}

Introduction: The specific diagnosis of influenza A infection makes it possible to control its spread, decreases the unnecessary use of antibiotics, clinical procedures and laboratory test, and allows early recognition of outbreaks. Different technologies are currently available in Chile for this purpose. Objective: The study presented here compares the sensitivity for influenza A virus detection of immunocromatography (RIDT), direct fluorescent antibodies-DFA and DFA with cytocentrifugation against the gold standard, RT-PCR. Material and Methods: In 175 nasal swab samples influenza A RIDT and RT-PCR were performed. Another 1689 nasal swab samples were tested by DFA and RT-PCR for influenza A. Finally, 29 nasal swab samples confirmed as Influenza A positive by RT-PCR were tested by DFA with cytocentrifugation. Results: The RIDT, DFA and DFA + cytocentrifugation sensitivity was $47,3 \%, 57,2 \%$ and $72,4 \%$, respectively. Discussion and Conclusion: Their lower cost and faster turnaround time when compared to PCR make RIDT and DFA the tests of choice in diagnostic laboratories in Chile. However, their low sensitivity and NPV, especially during low season, makes more sensitive diagnostic tools necessary to confirm the results. In our study cytocentrifugation increased DFA sensitivity from $57 \%$ to $72 \%$.

Key words: Influenza A, direct fluorescent assay, immunochromatography, cytocentrifugation, RT- PCR.

Palabras clave: Influenza A, inmunofluorescencia directa, inmunocromatografía, citocentrifugado.

\section{Introducción}

L os virus respiratorios se presentan con una incidencia variable durante el año, de carácter estacional, siendo influenza A uno de los relevantes en Chile durante la temporada de invierno ${ }^{1}$. El diagnóstico específico de influenza ha permitido controlar la diseminación de la enfermedad en la comunidad y en los hospitales, disminuir el uso de antimicrobianos, procedimientos clínicos y exámenes de laboratorio innecesarios, reducir la estadía hospitalaria, mejorar el manejo de pacientes de alto riesgo, guiar las decisiones de terapia antiviral e identificar rápidamente brotes ${ }^{2}$.

La inmunofluorescencia directa-IFD es ampliamente usada en hospitales regionales y laboratorios de referencia. Por otra parte, los exámenes inmunocromatográficos son fáciles de implementar y pueden ser usado en laboratorios locales a lo largo del país. La técnica de reacción de polimerasa en cadena en tiempo real con transcriptasa reversa (RPC-TR), incorporada en varios laboratorios chilenos durante la pandemia de la nueva Influenza A H1N1, es el nuevo estándar de oro para el diagnóstico específico de influenza $\mathrm{A}^{3,4}$, pero su costo y complejidad la hacen inviable en muchos laboratorios.

En el presente trabajo se evaluó la sensibilidad de una inmunocromatografía (test pack), IFD e IFD con citocentrifugado para influenza A contra RPC-TR.

\section{Objetivo}

Evaluar la sensibilidad diagnóstica de un examen inmunocromatográfico, de una IFD y de la IFD previa citocentrifugación, contra el estándar de oro (RPC-TR) en las infecciones por influenza A.

\section{Material y Métodos}

Se recolectaron 1.864 muestras mediante hisopado nasofaríngeo, las cuales se trasportaron refrigeradas $\left(0-4^{\circ} \mathrm{C}\right)$ en medio de transporte antigénico (MTA) y fueron procesadas en el Laboratorio Clínico de Clínica Alemana de Santiago dentro de las seis horas posteriores a su recolección.

Fueron estudiadas 175 muestras mediante inmunocro- 
matografía -test pack, kit comercial QuickVue ${ }^{\circledR}$ Influenza A + B (Quidel Corporation, San Diego, CA, USA)-, de acuerdo al protocolo del fabricante.

Por otra parte, 1.689 muestras fueron analizadas por IFD con kit comercial D3 Ultra $8^{\mathrm{TM}}$ DFA Respiratory Virus Screening \& Identification (Diagnostics Hybrids, Athens, OH, EEUU), de acuerdo al protocolo del fabricante.

Simultáneamente, todas las muestras fueron sometidas a RPC-TR en equipo Applied Biosystems 7500, según protocolo WHO-CDC para influenza A H1N1 (Primera revisión, abril 30, 2009), con sondas y partidores de Invitrogen $\left(\mathrm{N}^{\circ}\right.$ cat. A11400, Carlsbad, CA, EEUU) La enzima usada en la RPC-TR fue Superscript ${ }^{\mathrm{TM}}$ III Platinum ${ }^{\circledR}$ One-Step Quantitative RT- PCR System (Invitrogen, $\mathrm{N}^{\circ}$ cat. 11732-020, Carlsbad, CA, EEUU).

Para IFD con citocentrifugación previa, se tomaron todas las muestras recibidas entre el 30 de julio y 10 de agosto 2010 que resultaron positivas para influenza A H1N1 por RPC-TR (n: 29), se separó una alícuota de 150 $\mu l$ de muestra libre de mucosidad, se colocó en citotubo (Shandon single cytofunnel) y se centrifugó a $800 \mathrm{rpm}$ por 5 minutos en citocentrífuga Thermo Shandon. Tras fijar la lámina en acetona, se realizó inmunofluorescencia con kit comercial D3 Ultra $8^{\mathrm{TM}}$ DFA Respiratory Virus Screening \& Identification (Diagnostics Hybrids), de acuerdo al protocolo del fabricante.

Las muestras fueron solicitadas por los médicos tratantes como parte de la atención habitual de los pacientes y según los protocolos vigentes en Clínica Alemana, utilizando IFD para tamizaje rápido y RPC como resultado definitivo. Los costos de las IFD y RPC-TR fueron financiados por los pacientes y sus seguros ya que se trataba de una práctica clínica habitual. Los kits QuickVue (B) Influenza A + B fueron donados por Biomerieux Chile (Quidel Corporation, San Diego, CA, USA)

\section{Resultados}

En las 175 muestras en que se realizó inmunocromatografía y RPC-TR, 120 (68,5\%) fueron positivas y 55 $(31,5 \%)$ fueron negativas por RPC-TR. Comparadas con el total de muestras positivas por RPC-TR, la sensibilidad de la inmunocromatografía fue de $47,3 \%$; la especificidad fue de 99,2\%; su valor predictor negativo (VPN) fue de $80,4 \%$; su valor predictor positivo (VPP) fue de $96,3 \%$ (Tabla 1).

De 1.689 muestras en que se realizó IFD y RPC-TR, $752(44,5 \%)$ fueron positivas y $937(55,5 \%)$ fueron negativas por RPC-TR. Comparadas con el total de muestras positivas por RPC-TR, la sensibilidad de la IFD fue de $57,2 \%$; la especificidad de la IFD fue de $98,5 \%$; su VPN fue de 74,1\%; su VPP fue de 96,8\% (Tabla 2).
Tabla 1. Comparación de inmunocromatografía vs RPC para influenza A

\begin{tabular}{|llccc}
\multirow{7}{*}{ Test Pack } & & RPC-TR & \\
& & Positivo & Negativo & Total \\
& Positivo & 26 & 1 & 27 \\
& Negativo & 29 & 119 & 148 \\
& Total & 55 & 120 & 175 \\
\hline
\end{tabular}

Sensibilidad $=47 \%$. Especificidad $=99 \%$. VPP $=96 \%$. VPN $=80 \%$. RPC-TR: reacción de polimerasa en cadena en tiempo real con transcriptasa reversa.

\section{Tabla 2. Comparación de IFD vs RPC para influenza A}

\begin{tabular}{|lcccc|} 
& & & RPC-TR \\
& & Positivo & Negativo & Total \\
\multirow{3}{*}{ IFD } & Positivo & 430 & 14 & 444 \\
& Negativo & 322 & 923 & 1245 \\
& Total & 752 & 937 & 1689 \\
\hline
\end{tabular}

Sensibilidad $=57 \%$. Especificidad $=98 \%$. VPP $=97 \%$. VPN $=74 \%$. IFD: inmunofluorescencia directa. RPC-TR: reacción en cadena de polimerasa en tiempo real con transcriptasa reversa

\begin{tabular}{|c|c|c|}
\hline \multirow{4}{*}{$\mathrm{IFD}+\mathrm{CC}$} & & $\begin{array}{l}\text { RPC-TR } \\
\text { Positivo }\end{array}$ \\
\hline & Positivo & 21 \\
\hline & Negativo & 8 \\
\hline & Total & 29 \\
\hline \multicolumn{3}{|c|}{$\begin{array}{l}\text { Sensibilidad }=72 \% \text {. IFD }+ \text { CC: inmunofluorescencia directa con } \\
\text { citocentrifugado. RPC-TR: reacción de polimerasa en cadena en } \\
\text { tiempo real con transcriptasa reversa. }\end{array}$} \\
\hline
\end{tabular}

De las 29 muestras positivas mediante RPC-TR en que se realizó IFD con citocentrifugación previa, $21(72,4 \%)$ fueron positivas y $8(27,6 \%)$ negativas. (Tabla 3$)$.

\section{Discusión}

En la mayoría de los casos la infección por el virus influenza A se presenta como una patología leve, de baja mortalidad, pero como causa de importante morbilidad y hospitalizaciones ${ }^{3,5-7}$. Para influenza existe un tratamiento efectivo, por lo que el diagnóstico oportuno es necesario para iniciar tratamiento antiviral y como medida de control de propagación de la infección ${ }^{2}$. Si bien la aproximación diagnóstica puede en algunos casos realizarse mediante 
el cuadro clínico, la confirmación es por técnicas de laboratorio. La RPC-TR es el estándar de oro para el diagnóstico, pero dado su costo de implementación no se encuentra disponible en todos los centros del país.

En este estudio se analizó inmunocromatografía, la IFD y la IFD con citocentrifugación como alterativas para el diagnóstico. Tanto la inmunocromatografía como la IFD demostraron una buena especificidad, pero una sensibilidad moderada. Esto los hace una buena herramienta para momentos con alta prevalencia, pero su utilidad se ve disminuida fuera de estos períodos. Por venir listos para ser usados y entregar un resultado en 15 a 30 minutos, los Test Pack (inmunocromatografía) son aplicables en el lugar de atención del paciente para una prueba de tamizaje rápido, lo que les confiere una ventaja comparativa frente a la IFD en igualdad de sensibilidad.

Sin embargo, el presente estudio no comparó la IFD contra la inmunocromatografía directamente, siendo imposible recomendar uno por sobre el otro. Tampoco estudia el costo-beneficio de implementar alguno de los tests mencionados en los distintos servicios del país, por lo que es necesario nuevos estudios para determinar el impacto real de los distintos métodos diagnósticos en la práctica clínica y su beneficio económico.

Procesar las muestras mediante citocentrifugado previo a la IFD elevó la sensibilidad desde 57,2 a $72,4 \%$, transformando a la IFD en una herramienta más interesante para el diagnóstico de influenza A. Lamentablemente la metodología utilizada en este estudio no permite determinar especificidad o razón de verosimilitud (likelihood ratios), información valiosa que nos permitiría tener más información para recomendar o no este test.

El costo actual de la RPC-TR la hace prohibitiva para la mayoría de los servicios públicos en nuestro país, lo que obliga a buscar métodos alternativos de tamizaje y confirmación diagnóstica. Tanto el Test Pack como la IFD son insuficientes para realizar tamizaje durante las temporadas de baja prevalencia. Tampoco son útiles como método de confirmación diagnóstica.

El citocentrifugado es una técnica de mediana complejidad, que debe investigarse, dado su potencial aumento de la sensibilidad diagnóstica y VPN, siendo eventualmente de fácil implementación y menor costo que el actual estándar de oro ${ }^{3}$.

\section{Conclusión}

Tanto la inmunocromatografía como la IFD mostraron una sensibilidad moderada, pero su especificidad fue alta. El menor costo y tiempo de respuesta frente a la RPC-TR hacen de los test rápidos (IFD e inmunocromatografía) una interesante herramienta de tamizaje durante periodos de alta prevalencia. Sin embargo, su baja sensibilidad y VPN, mayor aún durante periodos de baja prevalencia, obliga a confirmar los resultados negativos.

El citocentrifugado mostró mejorar la sensibilidad de la IFD desde 57 a $72 \%$. Por ahora el menor costo y tiempo de respuesta frente a la RPC-TR hacen de este test una alternativa razonable para el diagnóstico de influenza A. Sin embargo, el rápido avance y masificación de los métodos moleculares, con su consecuente disminución en costos y tiempo, ponen en duda los beneficios del citocentrifugado más IFD versus los métodos moleculares en un futuro cercano.

Agradecimientos. A Bioimerieux Chile, por la donación de kits QuickVue ${ }^{\circledR}$ Influenza A + B (Quidel Corporation, San Diego, CA, USA). A Patricia González, por su gestión y apoyo.

\section{Resumen}

Introducción: El diagnóstico específico de influenza permite controlar la diseminación de la enfermedad, disminuir el uso de antimicrobianos, procedimientos clínicos y exámenes, e identificar rápidamente brotes. Diferentes tecnologías están actualmente disponibles en Chile para este propósito. Objetivo: Comparar la sensibilidad diagnóstica para la infección por el virus influenza A de las técnicas inmunocromatografía, inmunofluorescencia directa-IFD e IFD con citocentrifugado contra el estándar de oro, RPC-TR. Materiales y Método: En 175 muestras de hisopado nasofaríngeo se realizó inmunocromatografía y RPC-TR para influenza A. Otras 1.689 muestras de hisopado nasofaríngeo fueron procesadas mediante IFD y RPC-TR para influenza A. Finalmente, en 29 muestras de hisopado nasofaríngeo, confirmadas positivas para influenza A mediante RPC-TR, se realizó IFD con citocentrifugado. Resultados: La sensibilidad de la inmunocromatografía, IFD e IFD + citocentrifugado fue de $47,3 \%, 57,2 \%$ y $72,4 \%$, respectivamente. Discusión y Conclusión: El menor costo y tiempo de respuesta de las técnicas rápidas (inmunocomatografía e IFD) en relación a la RPC-TR hacen que se mantengan como exámenes de rutina en los laboratorios diagnósticos del país. Sin embargo, su baja sensibilidad y VPN, especialmente durante períodos de baja prevalencia, obligaría a confirmar los resultados negativos con técnicas más sensibles. En nuestra comparación la citocentrifugación mejoró la sensibilidad de la IFD de 57\% a $72 \%$. 


\section{Referencias bibliográficas}

1.- Lagos Z R, Avendaño C L F, Levine M M Vigilancia sistemática de virus influenza, respiratorio sincicial, parainfluenza y adenovirus, en niños ambulatorios con infecciones respiratorias agudas. Rev Med Chile 1999; 127 (9): 1063-72.

2.- Barenfanger J, Drake C, Leon N, Mueller T, Troutt T. Clinical and financial benefits of rapid detection of respiratory viruses: an outcomes study. J Clin Microbiol 2000; 38 (8): 2824-8.
3.- Landry M L, Ferguson D. Cytospin-enhanced immunofluorescence and impact of sample quality on detection of novel swine origin (H1N1) influenza virus. J Clin Microbiol 2010; 48 (3): 957-9.

4.- Mahony J B. Detection of respiratory viruses by molecular methods. Clin Microbiol Rev 2008; 21 (4): 716-47.

5.- Chowell G, Bertozzi S M, Colchero M A, López-Gatell H, Alpuche-Aranda C, Hernández M, et al. Severe respiratory disease concurrent with the circulation of H1N1 influenza. N Eng1 J Med 2009; 361 (7): 674-9.

6.- Jamieson D J, Honein M A, Rasmussen S A, Williams J L, Swerdlow D L, Biggerstaff M S, et al. H1N1 2009 influenza virus infection during pregnancy in the USA. Lancet 2009; 374 (9688): 451-8

7.- Pérez-Padilla R, la Rosa-Zamboni D de, Ponce de Leon S, Hernandez M, QuiñonesFalconi F, Bautista E, et al. Pneumonia and respiratory failure from swine-origin influenza A (H1N1) in Mexico. N Engl J Med 2009; 361 (7): 680-9. 